\title{
Reducción Selectiva de las Corrientes de Neutro en Instalaciones Eléctricas Mediante el Uso de Compensadores Activos Paralelo
}

\author{
Nicolás Muñoz, Fernando Villada, y Jesús M. López \\ Fac. de Ingeniería, Depto Ing. Eléctrica, Grupo de Manejo Eficiente de la Energía - GIMEL, Universidad de \\ Antioquia, Calle 67 No. 53-108, Oficina 19-437, Medellín - Colombia. \\ (e-mail: nicolasm@udea.edu.co, fvillada@udea.edu.co, lezama@udea.edu.co)
}

Recibido Sep. 24, 2012; Aceptado Nov. 12, 2012; Versión final recibida Dic. 23, 2012

\begin{abstract}
Resumen
Se usa un compensador activo de potencia paralelo para la reducción de las corrientes que circulan por el neutro en instalaciones eléctricas trifásicas a cuatro hilos. Las corrientes que debe inyectar el compensador activo a la carga son deducidas obteniéndose expresiones para reducir de forma global y selectiva las corrientes del neutro. Se diferencian las corrientes provocadas por cargas lineales desequilibradas y las corrientes provocadas por cargas no lineales. Las corrientes que circulan por el neutro causan diversos problemas en los sistemas eléctricos de acuerdo a su naturaleza u origen. La reducción selectiva de la corriente del neutro permite abordar estos problemas, de acuerdo a la causa que los producen, de tal manera que es posible maximizar la eficiencia y confiabilidad de los sistemas eléctricos.
\end{abstract}

Palabras clave: compensador de potencia paralelo, corrientes de compensación, cargas desequilibradas, cargas no lineales

\section{Selective Reduction of Neutral Currents in Electrical Installations by means of Shunt Active Compensators}

\begin{abstract}
A shunt active power compensator is used for neutral current reduction in three-phase four-wire electric installations. The power compensator currents injected to loads are deduced. Expressions to reduce the neutral current are obtained for global and selective compensation. The currents caused by unbalanced linear loads and nonlinear loads are differentiated. The neutral currents cause several problems in electric systems according to its nature or origin. The selective neutral current reduction allows solving such problems according to the cause that produce them, so that it is also possible to maximize the efficiency and reliability of electrical systems.
\end{abstract}

Keywords: shunt active power compensator, compensation currents, unbalanced loads, non-linear loads 


\section{INTRODUCCIÓN}

La corriente que circula por el neutro en los sistemas eléctricos causa múltiples problemas técnicos del día a día que se deben resolver. Jou et al. (2012) estudian la situación en las que las corrientes del neutro ocasionan la sobrecarga del conductor del neutro, también proponen un método de solución. Dunn y Wells (1998) analizan la situación en que las corrientes del neutro provocan sobretensiones neutro-tierra, de tal forma que, las protecciones contra fallas a tierra pueden funcionar inapropiadamente. Gruzs (1990) analiza la problemática de las corrientes que circulan por el neutro y que causan inconvenientes en las salas de cómputo y cargas electrónicas sensibles. Singh et al. (2005) abordan la problemática de tener cargas desequilibradas en las redes de distribución provocando el incremento de las pérdidas de potencia por el conductor del neutro.

Existen diferentes topologías basadas en electrónica de potencia y controles para reducir la corriente que circula por el neutro. Jou et al. (2012) utilizan un transformador de secuencia cero y un inversor para reducir las componentes de corriente que circulan por el neutro. Zadeh y Farjah (2009) utilizan un compensador activo para reducir las corrientes no fundamentales que circulan por el neutro y que son causadas por cargas no lineales. Singh et al. (2010) analizan una amplia variedad de transformadores y conexiones para atrapar las corrientes que circulan por el neutro evitando que circulen hacia la red eléctrica. Sewan y Minsoo utilizan un filtro hibrido (inversor y transformador zig-zag) para eliminar las corrientes no lineales y que circulan por el neutro de los sistemas eléctricos.

En la literatura técnica se han reportado estrategias de compensación para reducir las corrientes ineficientes de la carga. Escobar et al (2007) utilizan un SAPC para reducir la potencia reactiva y la distorsión armónica cuando el sistema es alimentado con voltajes distorsionados y asimétricos. Realizan el cálculo matemático de las corrientes de compensación en las coordenadas $\alpha \beta \gamma$ en función de los voltajes de línea y de la conductancia del sistema. Los autores mencionan que la compensación falla cuando existen componentes de voltaje homopolar, por lo que para el correcto funcionamiento, estas deben ser eliminadas.

Herrera y Salmerón (2009) mejoran el procedimiento de cálculo de la teoría de la potencia instantánea reactiva y plantean dos estrategias de compensación. Las estrategias de compensación incluyen: 1) La obtención de factor de potencia unitario. 2) La compensación de corrientes no sinusoidales y desequilibradas. Las corrientes de compensación son calculadas en función de las potencias y voltajes en coordenadas $\alpha \beta \gamma$.

Orts et al (2008) realizan la compensación de potencias ineficientes basados la IEEE Std 1459 (2010) y reducen las corrientes ineficientes causadas por cargas no lineales, desequilibrios, y la presencia de cargas reactivas.

Si el cálculo de las corrientes de compensación incluye voltajes, potencias, y conductancias Escobar et al (2007), y Herrera y Salmerón (2009), las estrategias de compensación presentan problemas cuando los voltajes de alimentación son asimétricos o presentan distorsión armónica. En este artículo, se plantean las corrientes de compensación en función de las corrientes de la carga y no en función de voltajes, potencias y conductancias garantizando la compensación de las corrientes del neutro aun cuando los voltajes presenten asimetría o distorsión armónica. Además, en este artículo, se plantean las corrientes de compensación del neutro relacionándolas con efectos ineficientes en la carga (asimetría y distorsión). En la literatura técnica no se ha reportado tal distinción y la reducción de la corriente del neutro se realiza sin distinguir los efectos físicos que la provocan.

La contribución del artículo es el planteamiento de las corrientes de compensación global y selectiva para la reducción de las corrientes del neutro relacionando las corrientes ineficientes de la carga con los fenómenos que la producen. Las corrientes que circulan por el neutro son corrientes de secuencia cero $\left(\mathrm{I}_{1}^{0}\right.$ e $\left.\mathrm{I}_{\mathrm{H}}^{0}\right)$ y son ocasionadas generalmente por las cargas conectadas o usuarios finales. La corriente $\mathrm{I}_{1}^{0}$ se debe principalmente al desequilibrio de la carga, y la corriente $\mathrm{I}_{\mathrm{H}}^{0}$ es parte de la corriente no fundamental $\left(\mathrm{I}_{\mathrm{H}}\right.$ ) que demandan las cargas no lineales. En este artículo se propone el uso de un compensador SAPC para la reducción selectiva de las corrientes $\mathrm{I}_{1}^{0}$ e $\mathrm{I}_{\mathrm{H}}^{0}$. Se proponen las corrientes de compensación que deben de ser inyectadas para eliminar cada corriente, también se presentan las ecuaciones para reducir de forma global las corrientes que circulan por el neutro. Ensayos experimentales son realizados para verificar las ecuaciones propuestas. 


\section{EL COMPENSADOR ACTIVO DE POTENCIA PARALELO (SAPC)}

Un SAPC es un dispositivo electrónico usado como fuente de corriente controlada, para suministrar las corrientes ineficientes que está demandando la carga evitando que circulen por la red. La conexión del SAPC se realiza en el pcc (punto de conexión común) quedando en paralelo con la carga en la red de distribución. EI SAPC suministra a la carga las corrientes ineficientes, por lo que el sistema eléctrico de potencia dejará de entregarlas, evitándose así los efectos adversos sobre el sistema eléctrico y mejorando la eficiencia del mismo. El diagrama unifilar de conexión de un SAPC a la red se muestra en la figura 1.

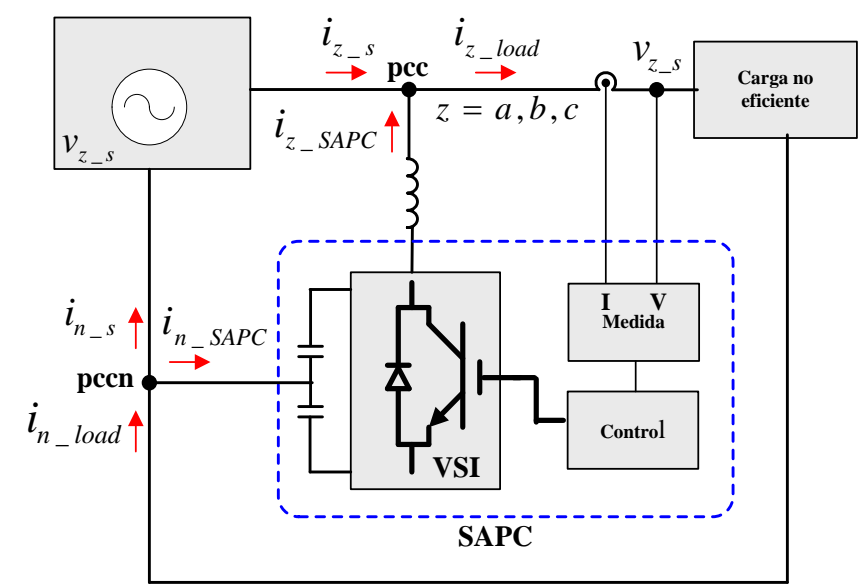

Fig 1. Diagrama de conexión de un SAPC.

El principal componente de los SAPC's es el inversor de voltaje (Voltage Source Inverter VSI). El inversor convierte tensión continua en alterna disponiendo en su entrada de un bus de tensión continua y en su salida de un bus de tensión alterna. La mayor parte de los VSI's comerciales contienen tres ramas de transistores IGBT's, de tal forma, que es posible inyectar por estas ramas (fases $a, b$, y $c$ ) las corrientes ineficientes que demanda la carga liberando al sistema eléctrico de entregar estas corrientes.

En la figura (1), existe una fuente de tensión $v_{z_{s}}$ que alimenta una carga no eficiente que demanda las

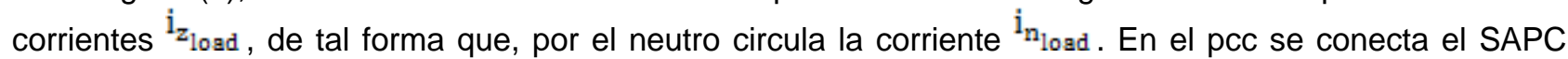
inyectando a la carga las corrientes ${ }^{i_{\text {SAPC }}}$ por las fases $a, b$, y $c$; por el neutro del SAPC circula la corriente

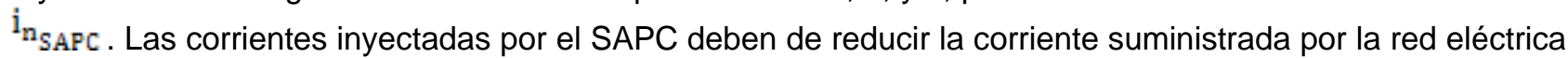
$\left(\hat{i}_{z_{g}}\right)$ y su corriente de neutro $\left(i_{n_{g}}\right)$. La reducción de la corriente $\mathfrak{i}_{n_{g}}$ depende de las corrientes de compensación que debe inyectar el SAPC. En el pccn confluyen las corrientes del neutro de la red eléctrica, del SAPC, y de la carga. A continuación se deducirán las corrientes de compensación para reducir las corrientes que circulan por el neutro.

\section{CORRIENTES DE COMPENSACIÓN PARA LA REDUCCIÓN DE LAS CORRIENTES QUE CIRCULAN POR EL NEUTRO}

Las corrientes que circulan por el neutro son principalmente corrientes de secuencia cero aunque también pueden existir por este conductor componentes de corriente de secuencia positiva y de secuencia negativa. Las corrientes de secuencia cero se pueden dividir en corrientes fundamentales de secuencia cero $\left(\mathrm{I}_{1}^{0}\right)$ y corrientes no fundamentales de secuencia cero $\left({ }_{H}^{0}\right)$. La corriente $I_{1}^{0}$ se debe principalmente al desequilibrio de la carga lineal y se puede obtener si se le realiza la transformada de Fortescue a las corrientes fundamentales de cada fase que demanda la carga $\left(\mathrm{I}_{\mathrm{a} 1}, \mathrm{I}_{\mathrm{b} 1}, \mathrm{I}_{\mathrm{c} 1}\right)$. En un sistema trifásico a 4 hilos, a cada fase se le atribuye la demanda de $\mathrm{I}_{1}^{0}$, mientras que por el neutro circula una corriente de valor $3 \mathrm{I}_{1}^{0}$. La corriente $\mathrm{I}_{\mathrm{H}}^{0}$ es propia de las cargas no lineales equilibradas, cuando producen armónicos de corriente de orden $\mathrm{h}=3 n$ con $\mathrm{n}=1,2,3, \ldots, \infty$ (armónicos de secuencia cero) y se encuentran en fase. En los sistemas trifásicos a 4 hilos, ${ }_{\mathrm{H}}^{0}$ circula por cada fase, mientras que por el neutro se suman estas corrientes y circula una corriente de valor $3 I_{\mathrm{H}}^{0}$. La aparición de armónicos de corriente de secuencia positiva o negativa desequilibrados $\left(\mathrm{I}_{\mathrm{H}}^{\frac{ \pm}{\mathrm{H}}}\right)$ también contribuye a la circulación de corriente por el neutro, esta situación no suele ser usual pero puede presentarse. Las corrientes que circulan por el neutro son expresadas en términos de las corrientes instantáneas como sigue: 
$\mathrm{i}_{\mathrm{n}_{\text {logd }}}=\mathrm{i}_{\mathrm{n}_{\text {load }}}^{0}+\mathrm{i}_{\mathrm{nH}_{\text {load }}}$

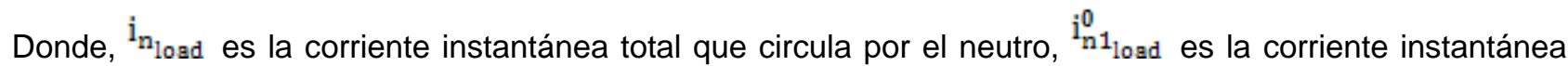
fundamental de secuencia cero del neutro, $y \mathrm{i}_{\mathrm{nH}_{\text {losd }}}$ es la corriente instantánea no fundamental del neutro.

La corriente $\hat{\mathrm{i}}_{\mathrm{nH}} \mathrm{Hogd}_{\text {log }}$ puede dividirse a su vez en la corriente instantánea no fundamental de secuencia cero del neutro $\left({ }^{1}{ }^{0} H_{l o g d}\right)$, y en la corriente instantánea no fundamental de secuencia positiva y/o negativa desequilibrada del neutro $\left({ }^{\mathrm{i}} \mathrm{nH}_{\text {load }}^{ \pm}\right)$:

$\mathrm{i}_{\mathrm{nH}} \mathrm{H}_{\text {logd }}=\mathrm{i}_{\mathrm{nH}}^{0}+\mathrm{i}_{\mathrm{n} \mathrm{H}_{\text {load }}}^{ \pm}$

La corriente ${ }^{i_{\mathrm{n}_{\text {load }}}}$ puede ser medida directamente con un sensor de corriente en el conductor de neutro de la carga, o puede obtenerse indirectamente a través de la medida de la corriente de carga de las tres fases

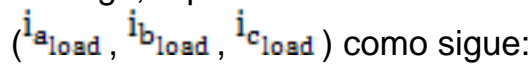

$\mathrm{i}_{\mathrm{n}_{\text {load }}}=\mathrm{i}_{\mathrm{alogd}_{\text {log }}}+\mathrm{i}_{\mathrm{b}_{\text {logd }}}+\mathrm{i}_{\mathrm{c}_{\text {logd }}}$

Con la expresión (3), las componentes de corriente fundamental de secuencia positiva y negativa se anulan, mientras que las componentes de corriente que están en fase se suman. La ecuación (3) contiene todas las componentes de corriente que circulan por el neutro: contiene a la corriente fundamental de secuencia cero $\left(I_{1}^{0}\right)$, a las corrientes no fundamentales de secuencia cero $\left({ }_{H}^{0}\right)$ equilibradas, e inclusive a las corrientes no fundamentales de secuencia positiva y negativa desequilibradas $\left(\mathrm{I}_{\mathrm{H}}^{+}, \mathrm{e} \mathrm{I}_{\mathrm{H}}^{-}\right)$. La corriente que debe generar el SAPC en su salida ( ${ }^{i_{z n_{S A P C}}}$ ) para eliminar de forma global las corrientes del neutro está dada por:

$\mathrm{i}_{\text {zn }}=\frac{1}{3} i_{n_{\text {logd }}}$

A continuación se deducirán las corrientes de compensación para cada corriente que circula por el neutro. Se deducirá la corriente instantánea fundamental causada por cargas lineales desequilibradas y se deducirá la corriente instantánea no fundamental causada por cargas no lineales.

Cálculo de la corriente de compensación selectiva para la reducción de la corriente instantánea fundamental que circula por el neutro.

La corriente instantánea fundamental que circula por el neutro $\left(\mathrm{i}_{\mathrm{m}}^{\mathrm{i}} \mathrm{losd}_{\mathrm{g}}\right)$ se obtiene a partir de la medida 0 cálculo de la corriente del neutro ( $\left.{ }^{\mathfrak{i}_{n_{\text {load }}}}\right)$. Se le debe aplicar a ${ }^{\mathrm{i}_{\mathrm{n}_{\text {logd }}}}$ la Transformada Discreta de Fourier (DFT por sus siglas en inglés). Debido a que por el neutro sólo circulan componentes de secuencia cero (sin

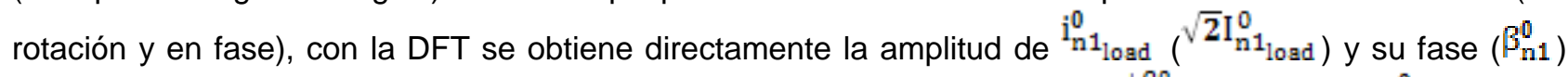
con respecto a la tensión fundamental de secuencia positiva $\left(V_{1}^{+}\right)\left(\theta_{\mathrm{m} 1}^{0}=\alpha_{1}^{+\beta}\right)$. El término ${ }^{I_{\mathrm{m}}^{0}}{ }_{\text {load }}$ es el valor rms de la corriente fundamental de secuencia cero que circula por la carga. $\mathrm{i}_{\mathrm{n}_{\mathrm{log}}}^{0}$ puede escribirse como sigue:

$\mathrm{i}_{\mathrm{n} 1_{\text {logd }}}^{0}=\sqrt{2} \mathrm{I}_{\mathrm{n} 1_{\text {logd }}}^{0} \operatorname{sen}\left(\omega_{1} t+\theta_{\mathrm{n} 1}^{0}\right)$

Si se dispone de un SAPC con un inversor de 4 ramas (ramas para las fases a, b, c y el neutro), es posible utilizar directamente la expresión (5) para calcular las corrientes de compensación a inyectar por la rama del neutro. Sin embargo, debido a que la configuración de SAPC implementada en el laboratorio contiene un inversor de 3 ramas, se debe obtener una corriente instantánea para cada fase activa del inversor y que retorne por el neutro $\left({ }^{i}{ }_{\operatorname{zn} 1}^{0} 1_{\text {SAPC }}\right)$, esta corriente puede calcularse como sigue:

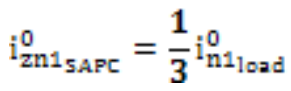


Cálculo de la corriente de compensación selectiva para la reducción de la corriente instantánea no fundamental que circula por el neutro.

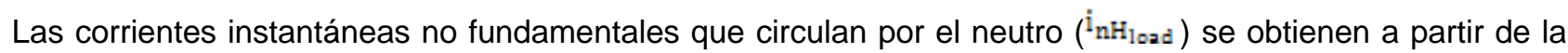

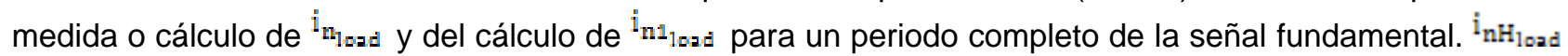
contiene todas las componentes de corriente no fundamental que circulan por el neutro y está dada por:

$\mathrm{i}_{\mathrm{nH}} \mathrm{H}_{\text {load }}=\mathrm{i}_{\mathrm{n}_{\text {lord }}}-\mathrm{i}_{\mathrm{mi} 1_{\text {load }}}$

La ecuación (7) sirve si se dispone de un SAPC con un inversor de 4 ramas, para el SAPC de 3 ramas implementado en el laboratorio, se debe obtener una corriente instantánea para cada fase activa del SAPC y que retorne por el neutro $\left({ }^{i_{\text {znH }}}\right.$ SAFc $\left._{\text {S }}\right)$, esta corriente puede calcularse como sigue:

$i_{\text {znH }}=\frac{1}{3} i_{\text {nAPC }} H_{\text {load }}$

La expresión (8) sirve para eliminar las componentes armónicas de corriente equilibrada y de secuencia cero que circulan por el neutro $\left({ }_{\mathrm{nH}}^{0}\right)$, sirviendo también para eliminar las componentes armónicas de corriente desequilibrada de secuencia positiva y negativa que circulan por el neutro ( $\left.{ }_{\mathrm{nH}}^{+}, \mathrm{e} \mathrm{I}_{\mathrm{nH}}^{-}\right)$.

Para reducir de forma selectiva las componentes de corriente armónica que circulan por el neutro también

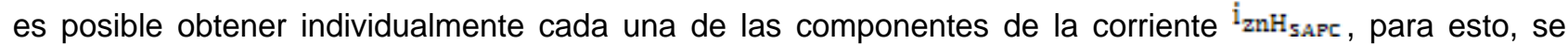
requiere del cálculo de la DFT para todas las componentes armónicas y su descomposición mediante la transformada de Stokvis-Fortescue. Por su alto costo computacional este algoritmo no fue implementado en el prototipo de laboratorio.

\section{RESULTADOS EXPERIMENTALES}

En esta sección se muestran los resultados experimentales obtenidos mediante la implementación de un SAPC en el laboratorio. La arquitectura del hardware del SAPC puede verse en la figura 2 y consiste básicamente en un inversor VSI de tres ramas (SKiiP 342GD120-314CTV) que inyecta en las terminales $A, B$, y $C$ las corrientes ineficiente que demanda la carga. El inversor es controlado mediante el DSP (TMS320F2812) que requiere la medida de los voltajes, corrientes de la carga, tensión en el bus DC, y corrientes de compensación a la salida del SAPC. Las señales de control y medida son acondicionadas mediante placas de adaptación de la medida, placas de adaptación señales de los PWM, y placas de adaptación de los sensores de protección del inversor. EI SAPC es conectado al pcc mediante contactores y resistencias de precarga, las señales de los contactores son llevadas a una placa de control con opto-triac's para su acondicionamiento. Las inductancias a la salida del inversor son utilizadas para reducir el efecto de los picos de corriente debido a la conmutación de los IGBT's del inversor. El filtro EMI es utilizado para filtrar las componentes de corriente de alta frecuencia y evitar que fluyan hacia la red eléctrica. El filtro EMI y las inductancias de salida se deben utilizar para reducir el ruido y satisfacer los limites máximos de EMI en normas internacionales Mainali et al (2008). Se utiliza un interruptor general diferencial y termomagnético como protección del sistema ante fallos. El equipo de medida es el osciloscopio digital Lecroy wavejet 324.

El diseño de la etapa de potencia debe incluir:

1) La capacidad de los condensadores del bus $D C\left(C_{1}\right.$ y $C_{2}$ de la figura (2)): Se debe considerar el caso más desfavorable que es una carga desequilibrada de secuencia negativa. Bajo esta condición, el rizado del bus DC tiene una frecuencia igual al doble de la frecuencia de la red eléctrica, la capacitancia puede calcularse mediante la expresión (9) Bojrup (1999). Donde $S$ (1 Kva) es la potencia aparente nominal del SAPC, $\omega_{1}$ $\left(2 \pi^{*} 50\right)$ es la frecuencia fundamental de la red en el pcc, $V_{\mathrm{dc}}(800 \mathrm{~V})$ es la tensión máxima que puede soportar el bus $D C$ y $\Delta V_{\text {de }}(1 \mathrm{~V})$ es el máximo rizado permitido en el bus dc. Para los anteriores valores $C$ debe ser mayor a $3.9 \mathrm{mf}$, se eligió el valor de $\mathrm{C}=4.7 \mathrm{mf}$.

$C>\frac{S}{2 \omega_{1} V_{d c} \Delta V_{d c}}$

2) La tensión nominal del bus DC: La tensión del bus debe ser lo más bajo posible para minimizar las pérdidas por conmutación en los IGBT's aunque suele ponerse entre un valor de $10 \%$ o $15 \%$ superior a la 
tensión natural del bus (Cuando el inversor funciona como rectificador con los IGBT's desconectados). Para el control de la corriente del lado de alterna, la tensión de salida del inversor debe ser, al menos igual, a la amplitud de la tensión fase-neutro de la red en el pcc admitiendo un valor entre $+10 \%$ y $-15 \%$ de la tensión nominal. Para la configuración del inversor ( 3 ramas y 4 hilos con bus dc repartido adoptado para la implementación del SAPC) y la estrategia de modulación PWM se consideró una tensión $30 \%$ mayor a la tensión de la red en el pcc (220 Vdc con 1 Vdc de rizado).

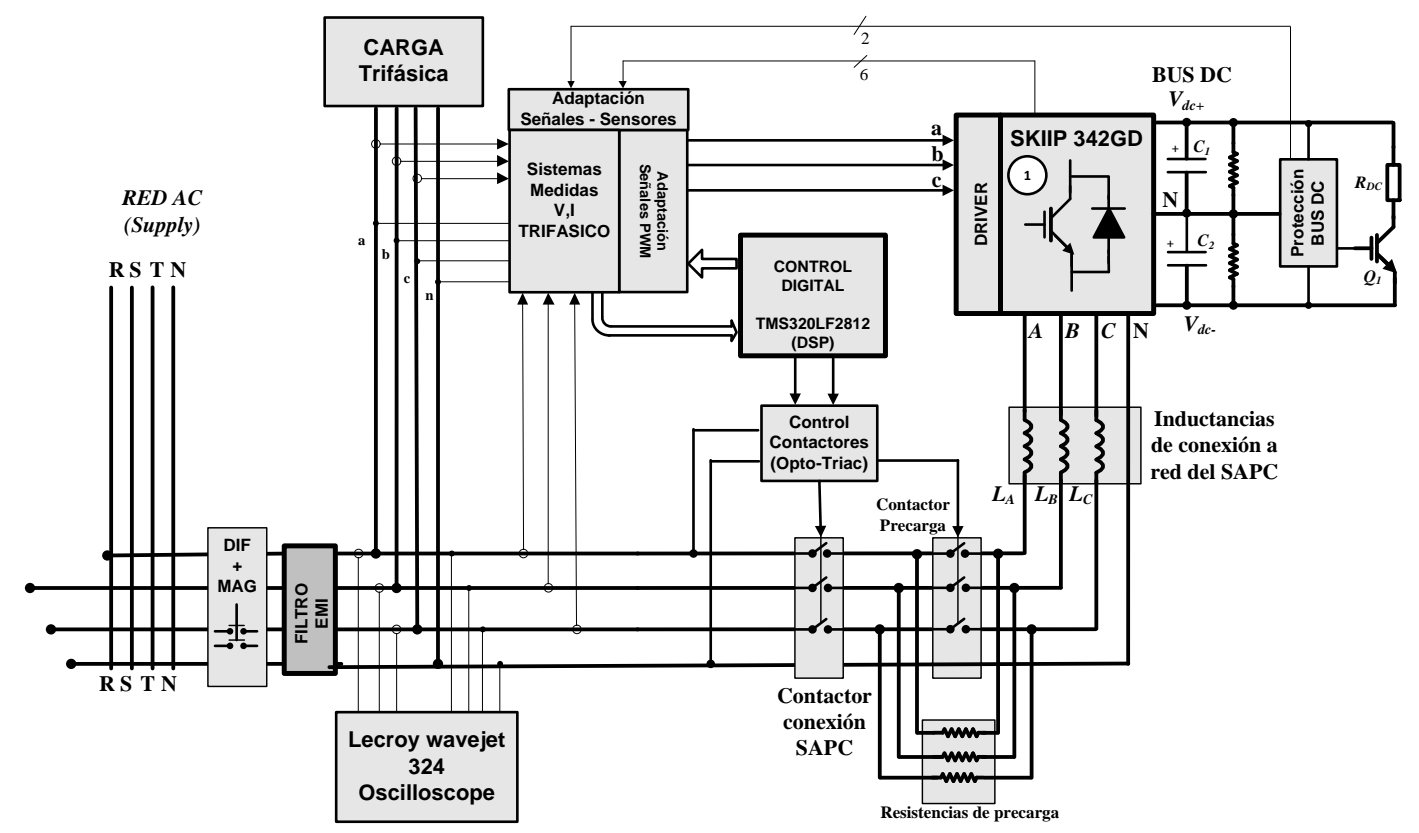

Fig 2. Arquitectura del hardware del prototipo de laboratorio.

3) El valor de la inductancia de las bobinas de salida: Las bobinas sirven para acoplar las dos fuentes de tensión (inversor DC y salida AC). Las inductancias son un filtro de primer orden pasabajos que reducen parte de los armónicos de alta frecuencia provocados por la conmutación de los IGBT's del inversor. Para su diseño se parte de la ecuación diferencial que rige el comportamiento de la tensión en la bobina. Se debe garantizar la tensión de $125 \mathrm{Vrms}$ en la carga y $220 \mathrm{Vdc}$ en el bus DC para una carga que demanda una corriente máxima de 10 A netamente inductiva Mohan et al (1995) y Bojrup (1999). Se eligió una bobina de $50 \mathrm{mH}$ que tiene una caída de tensión máxima de 15.7 Vrms. El voltaje a la salida del inversor cuando la carga es de 10 A y netamente inductiva es 140.7 Vrms (125+15.7), el valor pico es de 198.97 Vpico que no supera los 220 Vdc nominales del bus DC.

\section{El control del convertidor está compuesto por dos componentes principales:}

1) El control de corriente: Un SAPC es una fuente de corriente controlada y requiere una estrategia de control con realimentación de corriente, la corriente en la salida del convertidor debe seguir la corriente de referencia deseada. La corriente de referencia deseada para la compensación global de la corriente del neutro es la expresión (4), para la reducción de las componentes fundamentales es la expresión (6), para la reducción de las componentes no fundamentales es la expresión (8). El control de corriente debe garantizar el control de la forma de onda con gran precisión incluyendo variaciones en la carga, la protección contra picos de corriente y sobrecarga, y buena respuesta dinámica. En el SAPC fue implementado un regulador PI PWM, una recopilación de trabajos acerca de los métodos de control de corriente para convertidores PWM se encuentra Kazmierkowski y Malesani (1998).

2) El control del bus DC: Para el efectivo control de corriente, el SAPC requiere una tensión constante en el bus DC. El rizado del bus DC depende de las corrientes que el SAPC debe inyectar a la carga. Si el SAPC inyecta corrientes que causan flujos bidireccionales de potencia (potencias reactivas, potencias armónicas, y potencias de desequilibrio) el bus DC se descargará. Si el SAPC inyecta corrientes que causan flujos unidireccionales (pérdidas de potencia en el convertidor y potencias activas residuales que requiera la carga) el bus DC no se descargará. Una ampliación de la teoría de los flujos de potencia puede verse en Muñoz et al (2012). El control del bus DC depende de las corrientes de compensación inyectadas, para cargar el bus DC el SAPC debe absorber de la red eléctrica la corriente activa fundamental de secuencia positiva: 
$i_{z 1_{\text {SAPC }}^{+}}^{+}=-\sqrt{2} I_{z 1}^{+} \operatorname{sen}\left(\omega_{1} \mathrm{t}-\varphi_{z}\right)$

El valor de $\mathrm{I}_{z 1}^{+}$debe ser ajustado para cargar el bus DC y no permitir que disminuya más allá de su valor mínimo permitido o debe ser cero cuando el valor del bus DC es mayor que el valor máximo permitido (1 Vdc de rizado). Para las fases $z=a, b$, y $c ; \varphi_{z}$ toma valores de $0,-2 \pi / 3$, y $-4 \pi / 3$. El signo (-) indica que la corriente fluye desde la red de suministro hacia el SAPC. La corriente de la ecuación (11) debe ser sumada a la corriente de referencia del regulador de corriente PI del convertidor garantizando el suministro de las corrientes de compensación del neutro y la absorción de las corrientes que regulan la tensión del bus DC.

La carga utilizada para hacer las pruebas de laboratorio puede verse en la figura 3, se utiliza una carga que cause problemas de calidad de la potencia tales como el desequilibrio de las corrientes, demanda de potencia reactiva, e inyección de armónicos de corriente hacia la red eléctrica mediante carga no lineal. Para provocar el desequilibrio de las corrientes de la carga en la fase a fue conectada una resistencia $\mathrm{R} \_$load de $65.9 \Omega$. Para provocar la demanda de potencia reactiva en las fases $a, b$, y $c$ fueron conectados transformadores de $125 / 24 \mathrm{~V}$ con carga inductiva. El devanado primario se conecta a la tensión de $125 \mathrm{~V}$, mientras que en el devanado secundario se conectan como carga bobinas ( $L$ load=2.48 $\mathrm{mH}$ ). El transformador con carga inductiva es usado para aumentar la impedancia inductiva incrementando la demanda de potencia reactiva demandada por la carga. La carga no lineal en cada fase fue implementada utilizando un rectificador con carga $\mathrm{R} / / \mathrm{C}(\mathrm{L}=5 \mathrm{mH}, \mathrm{C}=2200 \mu \mathrm{F}, \mathrm{Rdc}=100 \Omega)$.

$125 \mathrm{~V}$

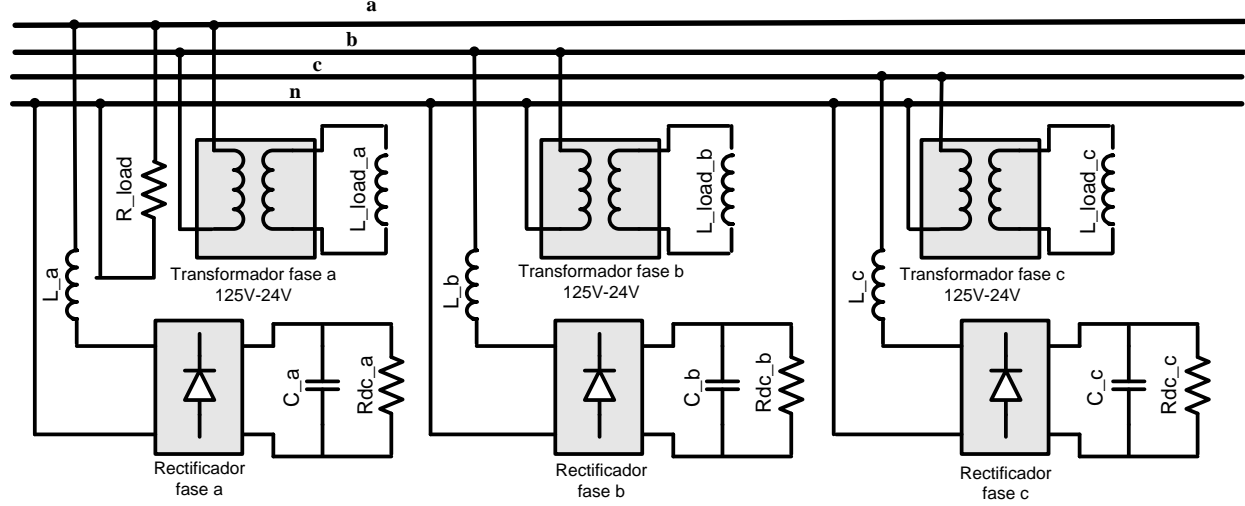

Fig 3. Carga trifásica utilizada en las pruebas de laboratorio.

El sistema trifásico de tensiones de alimentación senoidales y equilibradas $(125 \mathrm{~V} / 50 \mathrm{~Hz})$ fueron medidas en el pcc y sus formas de onda se muestran en la figura 4 (a). Las formas de onda de las corrientes de la carga

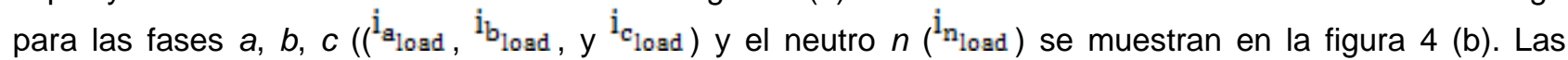
corrientes de la carga son las corrientes que entrega la red eléctrica antes de la compensación y son tomadas como referencia para evaluar las estrategias de compensación.

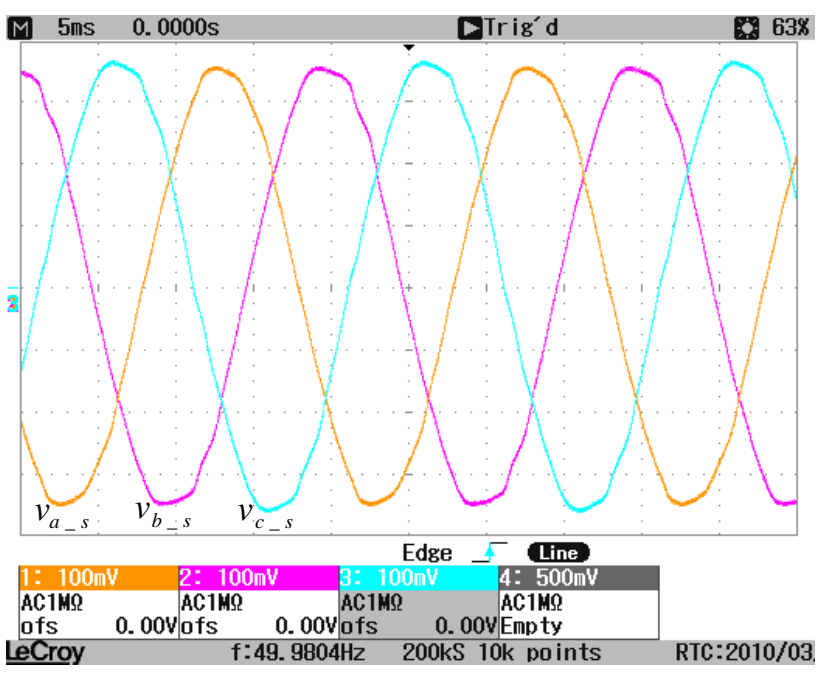

(a)

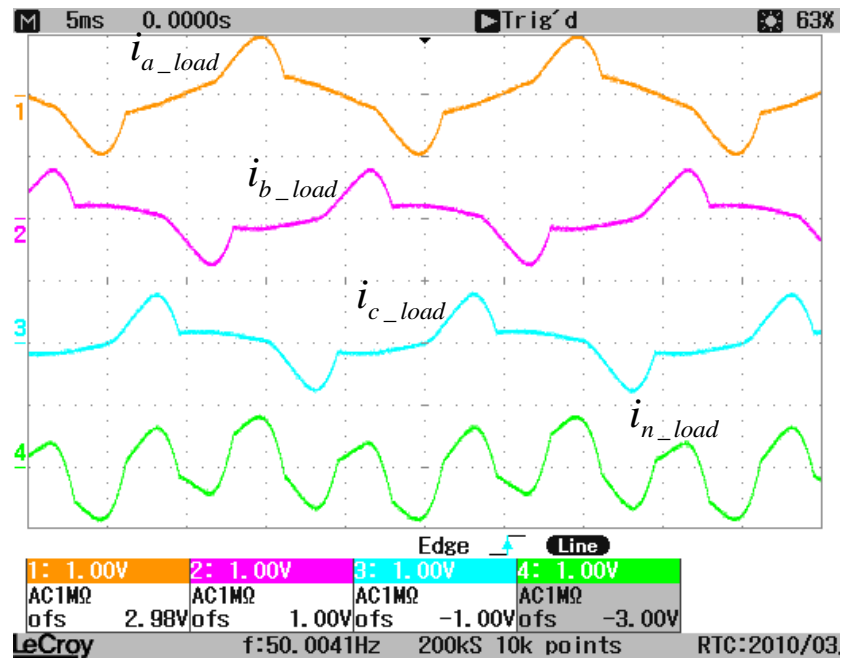

(b)

Fig 4. (a) Tensiones en el pcc. (b) Corrientes demandadas por la carga. 


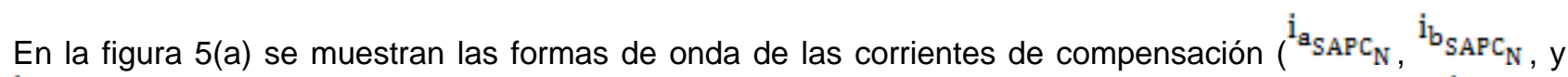
$\mathrm{i}_{\mathrm{S}_{\mathrm{SAPC}}}$ ) que son inyectadas por las fases $a, b, \mathrm{y} c$ a la carga (ecuación (4)). Las corrientes ${ }_{\mathrm{a}_{\mathrm{SAPC}}} \mathrm{I}_{\mathrm{N}}$, $\mathrm{i}_{\mathrm{b}_{S A P C}}, y{ }_{\mathrm{C}_{\mathrm{SAPC}}}$ son iguales y al sumarse en el pccn provocan la circulación de ${ }_{\mathrm{n}_{\text {SAPC }}}$ entre el SAPC y la carga reduciendo la corriente del neutro entre la red eléctrica y el SAPC. En la figura 5 (b) se muestran las corrientes que suministra la red durante la conexión del SAPC $\left({ }^{i_{a_{g}}},{ }_{b_{s}}, y{ }^{i_{c_{g}}}\right)$, como puede verse su forma

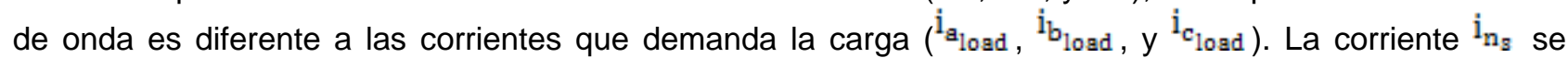

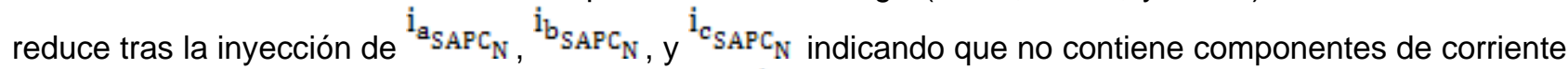
de baja frecuencia. El rizado que puede notarse en $\mathrm{i}_{n_{g}}$ se debe a las componentes de alta frecuencia por la conmutación de los IGBT's del inversor.

En la figura 6(a) se muestran las corrientes de compensación de las fases a, b, y c (ecuación (6)). La ecuación (6) indica que las corrientes de compensación deben ser iguales, mas sin embargo en la figura 6 (a) puede verse que sus amplitudes no son iguales y se encuentran desfasadas, esto se debe a que el SAPC simultáneamente está cargando el bus DC con corrientes fundamentales de secuencia directa provenientes de la red eléctrica (ecuación (11)). En la figura 6 (b) se muestran las corrientes que suministra la red durante la conexión del SAPC cuando se inyectan las corrientes de compensación de la ecuación (6). Las corrientes fundamentales que circulan por el neutro y que son causadas por el desequilibrio de la carga son reducidas. Si se comparan las corrientes de la carga (figura 4(b)) y las corrientes de la red de suministro (figura 6(b)) puede notarse que la inyección de las corrientes de compensación equilibra las corrientes de la red de suministro (igual valor pico-pico en las corrientes de la figura $6(b)$ ).

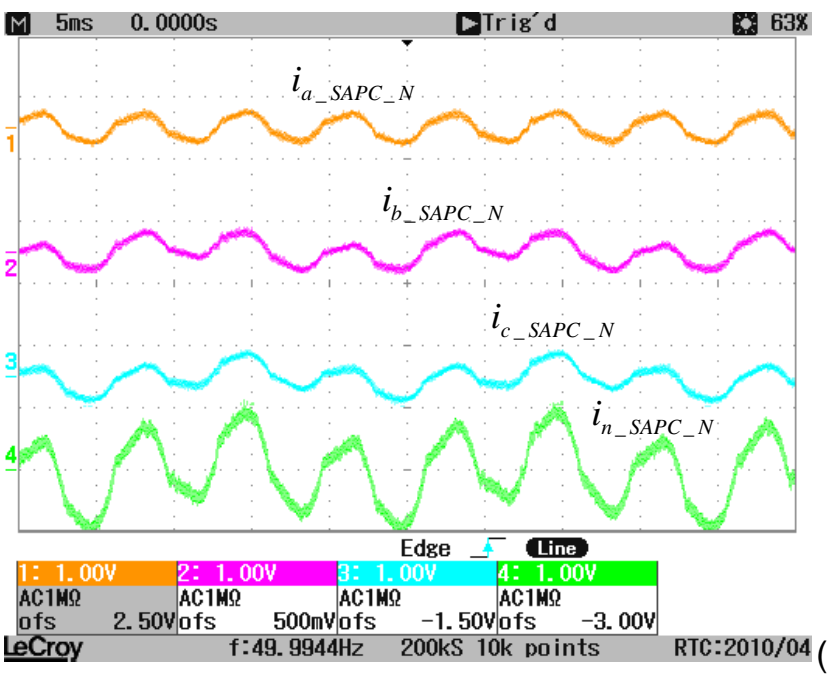

a)

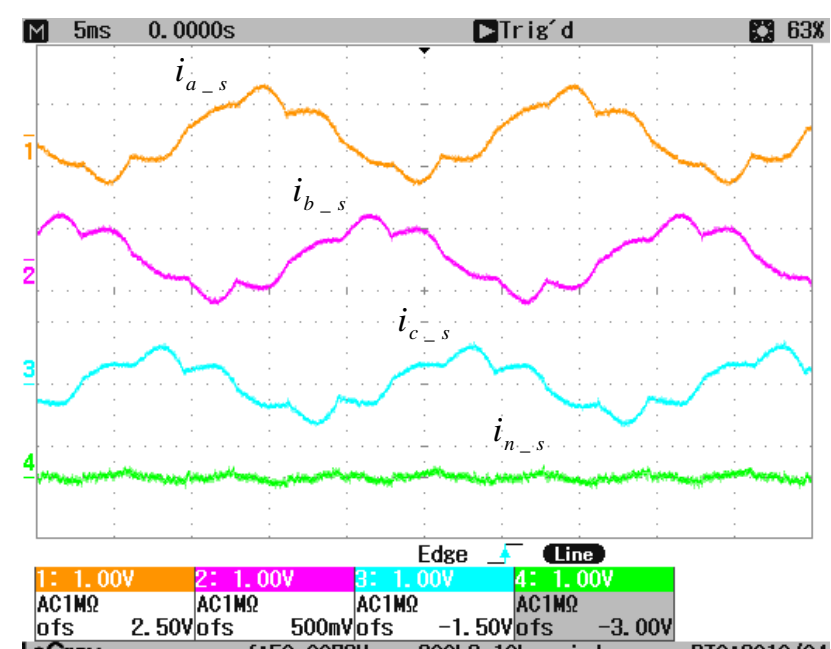

b)

Fig 5. (a) Corrientes de compensación (Ecuación (4)). (b) Corrientes de la red eléctrica.

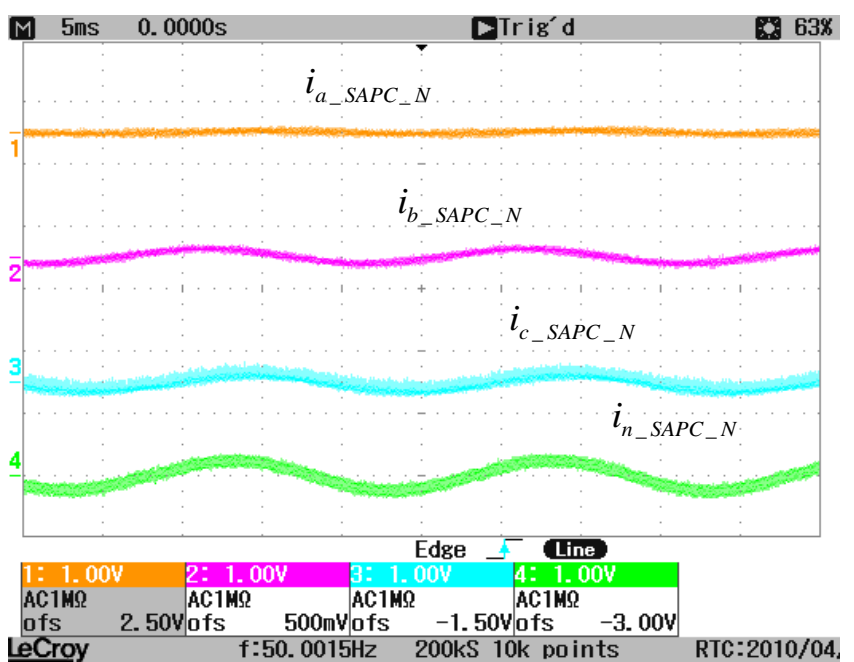

(a)

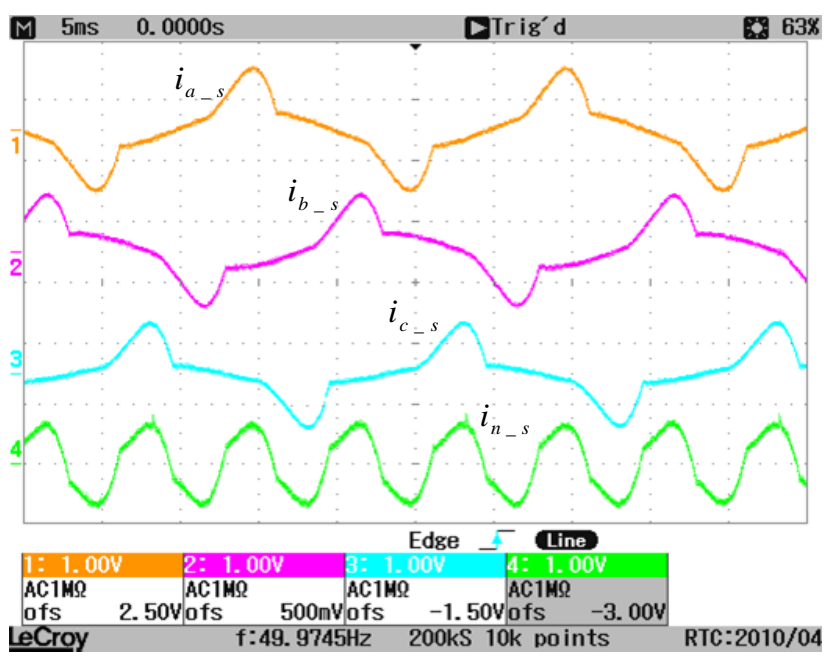

(b)

Fig 6. (a) Corrientes de compensación (Ecuación (6)). (b) Corrientes de la red eléctrica. 
En la figura 7 (a) se muestran las corrientes de compensación no fundamentales de las fases $a, b, y c$ (ecuación (8)). En la figura 7(b) se muestran las corrientes que suministra la red durante la conexión del SAPC cuando se inyectan las corrientes de compensación de la ecuación (8). Las corrientes no fundamentales que circulan por el neutro y que son causadas por la conexión de cargas no lineales son reducidas. Si se comparan las corrientes de la carga (figura 4(b)) y las corrientes de la red de suministro (figura 7(b)) puede notarse que la inyección de las corrientes de compensación provoca cambios en la forma de onda de las corrientes de las fases, también puede notarse que las corrientes que circulan por el neutro están compuestas por componentes fundamentales. Tras la compensación las corrientes no fundamentales no son reducidas y se demuestra que es posible reducir de forma selectiva las corrientes que circulan por el neutro.

En la tabla (1) se muestran las corrientes rms de las fases $a, b, c$, y neutro $\left(\mathrm{I}_{\mathrm{a}_{g}}, \mathrm{I}_{\mathrm{b}_{g}}, \mathrm{I}_{\mathrm{c}_{g}}, \mathrm{I}_{\mathrm{n}_{\mathrm{g}}}\right)$ para la carga conectada y en la red eléctrica tras realizar las diferentes estrategias de compensación. También se muestran las corrientes $\mathrm{I}_{1}^{+}, \mathrm{I}_{1}^{-}$, e $\mathrm{I}_{1}^{0}$ producto de realizar la transformada de Fortescue a las corrientes fundamentales de las fases $a, b, y$. Las corrientes $I_{n 1}$ e $I_{n H}$ corresponden a las corrientes fundamental y no fundamental que circulan por el neutro y que pueden ser obtenidas si a la corriente del neutro $I_{n_{s}}$ se le aplica la transformada discreta de Fourier. En la tabla (2) se muestran las potencias usando las tres estrategias de compensación. Las potencias fueron calculadas de acuerdo al procedimiento descrito en la IEEE Std 1459 (2010).

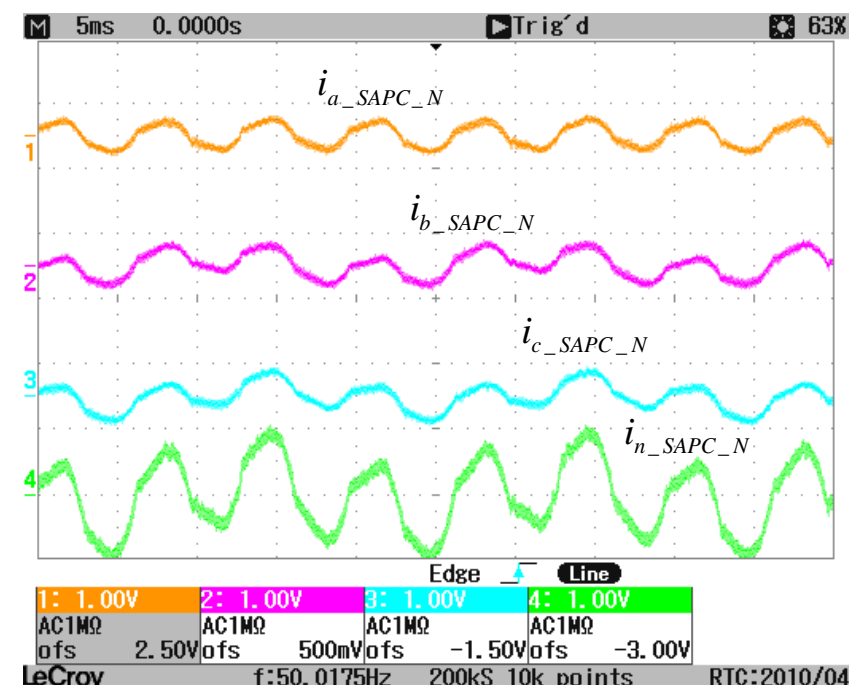

(a)

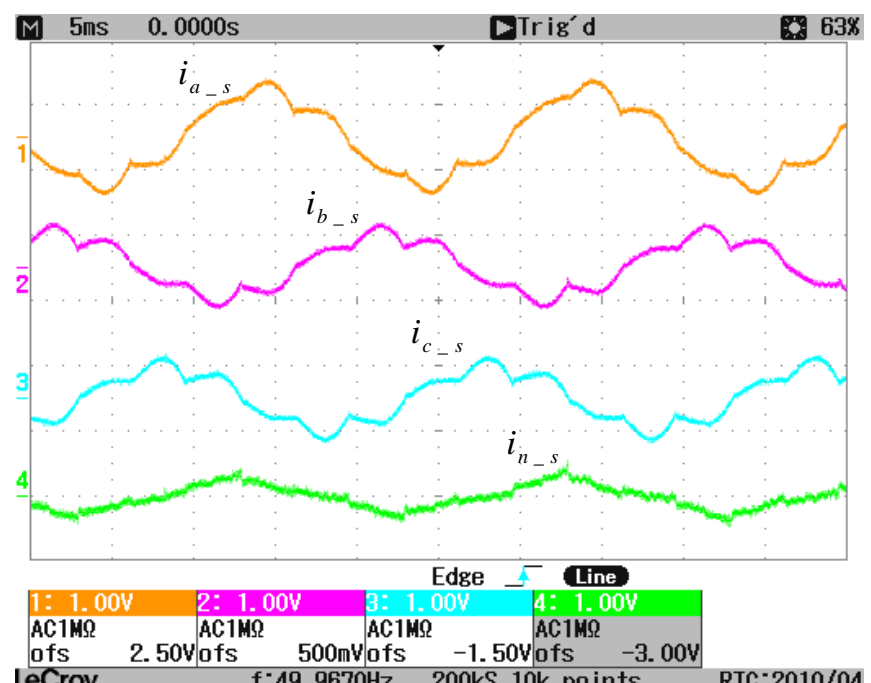

(b)

Fig 7. (a) Corrientes de compensación (Ecuación (8)). (b) Corrientes de la red eléctrica.

Tabla 1. Corrientes de la carga y de la red eléctrica en Amperios.

\begin{tabular}{|c|c|c|c|c|c|c|c|c|c|}
\hline Corrientes & $\mathrm{I}_{\mathrm{a}_{g}}$ & $\mathrm{I}_{\mathrm{b}_{s}}$ & $\mathrm{I}_{\mathrm{c}_{g}}$ & $\mathrm{I}_{\mathrm{n}_{s}}$ & $\mathrm{I}_{1}^{+}$ & $\mathrm{I}_{1}^{-}$ & $\mathrm{I}_{1}^{0}$ & $\mathrm{I}_{\mathrm{n} 1}$ & $\mathrm{I}_{\mathrm{nH}}$ \\
\hline Carga & 4.83 & 3.54 & 3.52 & 4.8 & 3.54 & 0.63 & 0.55 & 1.65 & 4.51 \\
\hline Ec. (4) & 4.59 & 4.13 & 3.58 & 0.46 & 3.98 & 0.64 & 0.03 & 0.1 & 0.45 \\
\hline Ec. (6) & 4.87 & 4.41 & 3.87 & 4.75 & 3.97 & 0.65 & 0.03 & 0.08 & 4.75 \\
\hline Ec. (8) & 5.24 & 3.69 & 3.62 & 1.78 & 4.07 & 0.65 & 0.58 & 1.72 & 0.45 \\
\hline
\end{tabular}

Tabla 2. Potencias de la carga y de la red eléctrica en Voltio-Amperios.

\begin{tabular}{|c|c|c|c|c|c|c|c|}
\hline Potencias & $\mathrm{S}_{\mathrm{e}}$ & $\mathrm{S}_{\mathrm{e} 1}$ & $\mathrm{~S}_{\mathrm{eH}}$ & $\mathrm{S}_{\mathrm{eN}}$ & $\mathrm{S}_{1}^{+}$ & $\mathrm{S}_{1}^{-}$ & $\mathrm{S}_{1}^{0}$ \\
\hline Carga & 1828.06 & 1409.70 & 23.26 & 1163.85 & 1327.18 & 1.95 & 1.716 \\
\hline Ec. (4) & 1548.49 & 1511.47 & 6.69 & 336.57 & 1492.14 & 1.98 & 0.09 \\
\hline Ec. (6) & 1745.08 & 1508.37 & 24.54 & 1170.26 & 1488.39 & 2.03 & 0.09 \\
\hline Ec. (8) & 1639.56 & 1555.24 & 6.63 & 333.67 & 1525.88 & 2.04 & 1.81 \\
\hline
\end{tabular}

En la fila 2 de la tabla 1 se muestran las corrientes que demanda la carga, al igual que la figura 4(b) puede notarse que las corrientes $I_{a_{s}}, I_{b_{s}}, I_{c_{s}}$ están desequilibradas ya que tienen valores rms diferentes. Las corrientes $\mathrm{I}_{1}^{0}=0.55 \mathrm{~A}$ e $\mathrm{I}_{\mathrm{n} 1}=1.65 \mathrm{~A}$ indican que la carga está desequilibrada, mientras que la corriente 
$\mathrm{I}_{\mathrm{nH}}=4.51 \mathrm{~A}$ indica la presencia de cargas no lineales. En la fila 2 de la tabla 2 también se puede verificar el desequilibrio de corrientes $\left(S_{1}^{0}=1.716 \mathrm{VA}\right.$, su valor es bajo porque depende de $\left.V_{1}^{0}=1.04 \mathrm{~V}\right)$. Las potencias $\mathrm{S}_{\mathrm{eN}}=1163.85 \mathrm{VA}$ y $\mathrm{S}_{\mathrm{eH}}=23.26 \mathrm{VA}$ indican que la carga es no lineal (el valor de $\mathrm{S}_{\mathrm{eH}}$ es bajo porque depende de $\mathrm{V}_{\mathrm{eH}}=\mathbf{2 . 5 \mathrm { V }}$ ).

En la fila 3 de la tabla 1 se muestran las corrientes de la red eléctrica cuando se realiza la compensación global (ecuación (4)), puede notarse que la diferencia entre las corrientes $I_{a_{s}}, I_{b_{s}}, I_{c_{s}}$ se reduce indicando que se han compensado las corrientes que causan el desequilibrio, también se reducen significativamente las componentes armónicas de corriente del neutro $\left(I_{\mathrm{nH}}=\mathbf{0 . 4 5 \mathrm { A }}\right), \mathrm{I}_{\mathrm{nH}}$ no se reduce totalmente debido a las corrientes armónicas de alta frecuencia que son provocadas por la conmutación de los IGBT's del SAPC. En la fila 3 de la tabla 2 puede notarse la reducción de $S_{1}^{0}=0.09 \mathrm{VA}, S_{\mathrm{eH}}=6.69 \mathrm{VA}$, y $\mathrm{S}_{\mathrm{eN}}=336.57 \mathrm{VA}$.

En la fila 4 de la tabla 1 se muestran las corrientes de la red eléctrica cuando se realiza la compensación de las corrientes fundamentales del neutro (ecuación (6)), puede notarse que las corrientes $\mathrm{I}_{1}^{0}$ e $\mathrm{I}_{\mathrm{n} 1}$ son prácticamente cero, mientras que la corriente $\mathrm{I}_{\mathrm{nH}}$ no es reducida. En la fila 4 de la tabla 2 sólo se reduce $\mathrm{S}_{1}^{0}=0.09 \mathrm{VA}$.

En la fila 5 de la tabla 1 se muestran las corrientes de la red eléctrica cuando se realiza la compensación de las corrientes no fundamentales del neutro (ecuación (8)), puede notarse que la corriente $\mathrm{I}_{\mathrm{nH}}$ es reducida, mientras que las corrientes $\mathrm{I}_{1}^{0}$ e $\mathrm{I}_{\mathrm{n} 1}$ no son reducidas. Con lo anterior se verifica experimentalmente las ecuaciones deducidas para realizar la compensación selectiva de las corrientes del neutro. En la fila 5 de la tabla 2 se reducen, $\mathrm{S}_{\mathrm{eH}}=6.63 \mathrm{VA}, \mathrm{y} \mathrm{S}_{\mathrm{eN}}=333.67 \mathrm{VA}$.

En la tabla 1 también puede notarse que la corriente $\mathrm{I}_{1}^{-}$permanece inalterada cuando se utilizan todas las estrategias de compensación, esto se debe a que las corrientes $\mathrm{I}_{1}^{-}$al sumarse en el pccn son cero no circulando por el neutro y las ecuaciones deducidas están basadas en las corrientes de secuencia cero que circulan por el neutro, es por esto que $\mathrm{S}_{1}^{-}$también permanece inalterada. Adicionalmente se puede notar un incremento de la corriente $\mathrm{I}_{1}^{+}$cuando se realiza la compensación de las corrientes del neutro, esto se debe a que el SAPC requiere potencia activa para cargar el bus de continua del inversor y para satisfacer las pérdidas de potencia producto de la conmutación de los IGBT's del inversor, es por esto que $S_{e 1}$ y $S_{1}^{+}$se incrementan.

\section{CONCLUSIONES}

El funcionamiento del SAPC propuesto y las corrientes de compensación es adecuado de tal forma que es posible reducir de forma global o forma selectiva las corrientes que circulan por el neutro. Las ecuaciones deducidas para la reducción de para de las corrientes del neutro fueron verificadas experimentalmente. Las corrientes de la ecuación (4) reducen de forma global la corriente que circula por el neutro, las corrientes de la ecuación (6) reducen de forma selectiva las corrientes fundamentales que circulan por el neutro, y las corrientes de la ecuación (8) reducen de forma selectiva las corrientes no fundamentales que circulan por el neutro.

Realizar la compensación selectiva de las corrientes del neutro contribuye con el mejoramiento de la calidad de la potencia mejorando la eficiencia y la confiabilidad de los sistemas eléctricos. La compensación selectiva de las corrientes del neutro permite aprovechar mejor la potencia del SAPC, de tal forma que es posible inyectar a la carga las corrientes ineficientes de acuerdo a los diferentes tipos de cargas existentes. Un posible trabajo futuro podría ser establecer los criterios de selectividad para la reducción de las corrientes que circulan por el neutro de los sistemas eléctricos.

El uso de los SAPC para la mejora de la calidad de la potencia y la eficiencia energética es viable, sin embargo, se debe tener en cuenta que los SAPC provocan pérdidas de potencia producto de su conexión. En términos generales, para el prototipo de laboratorio, al reducir las corrientes que circulan por neutro se logra mejorar la calidad de la potencia y la eficiencia energética de la red eléctrica, pero también se produce un leve incremento de $\mathrm{I}_{1}^{+}$y algunas componentes de corriente no fundamentales de alta frecuencia.

\section{AGRADECIMIENTOS}

Los autores agradecen a la Universidad de Antioquia (UdeA) por el apoyo de "Sos 2011-2012". 


\section{REFERENCIAS}

Bojrup, M., Advance Control of Active Filters in a Battery Charger Application, Licentiate Thesis TEIE-1021, ISBN: 91-88934-13-6. Dept. of Industrial Electrical Engineering and Automation, Lund Institute of Technology, Lund (Sweden), (1999).

Dunn, S.D., y F. Wells, Stray neutral current problems and analysis associated with multiple ATS generator installations, Industrial and Commercial Power Systems Technical Conference, 100-108, (1998).

Escobar, G., A.A. Valdez, R.E. Torres-Olguin, y M.F. Martinez-Montejano, A Model-Based Controller for A Three-Phase Four-Wire Shunt Active Filter With Compensation of Neutral Line Current, IEEE Transaction on Power Electronics, 22(6), 2261-2270, (2007)

Gruzs, T.M., A survey of neutral currents in three-phase computer power systems, IEEE Trans. Industry Applications, 26 (4), 719-725, (1990).

Herrera, R.S., y P. Salmerón, Instantaneous Reactive Power Theory: A Reference in the Nonlinear Loads Compensation, IEEE Transaction on Industrial Electronics, 56(6), 2015-2022, (2009)

IEEE Standard Definitions for the Measurement of Electric Power Quantities Under Sinusoidal, Nonsinusoidal, Balanced, or Unbalanced Conditions, IEEE Std 1459-2010, 1-40, (2010).

Jou, H.-L., K. Wu, y S. Xiao, Single-phase inverter-based neutral-current suppressor for attenuating neutral current of three-phase four-wire distribution power system, IET Generation Transmission and Distribution, 6 (6), 577-583, (2012).

Kazmierkowski, M. P., y L. Malesani, Special section on PWM current regulation, IEEE Trans. on Industrial Electronics, 45 (5), 689-835, (1998).

Mainali, K., R. Oruganti, K. Viswana, y S. Peng Ng, A Metric for Evaluating the EMI Spectra of Power Converters, IEEE Transaction on Power Electronics, 23 (4), 2075-2081, (2008).

Mohan, N., T.M. Undeland, y W.P. Robbins, Power Electronics, Converter, Applications and Design, John Wiley\&Sons, Inc., (1995).

Muñoz, N., F. Villada, y D.R. Cadavid, Análisis de los Flujos de Potencia de Sistemas Eléctricos y su Interpretación Física, Información Tecnológica, 23 (5), 57-68, (2012).

Orts-Grau, S., F.J. Gimeno-Sales, S. Seguí-Chilet, A. Abellan-García, M. Alcaniz, y R. Masot-Peris, Selective Shunt Active Power Compensator Applied in Four-Wire Electrical Systems Based on IEEE Std. 1459, IEEE Transaction on Power Delivery, 23 (4), 2563-2574, (2008).

Sewan, C., y M. Jang, Analysis and Control of a Single-Phase-Inverter-Zigzag-Transformer Hybrid NeutralCurrent Suppressor in Three-Phase Four-Wire Systems, IEEE Transaction on Industrial Electronics, 54 (4), 2201-2208, (2007).

Singh, B., J. Solanki, y V. Verma, Neural Network Based Control of DSTATCOM with Rating Reduction for Three-Phase Four-Wire System, International conference on Power Electronics and Drive Systems, 920925, (2005).

Singh, B., P. Jayaprakash, y D.P. Kothari, Magnetics for neutral current compensation in three-phase fourwire distribution system, Int Conf on Power Electronics, Drives and Energy Systems (PEDES), 1-7, (2010).

Zadeh, J.K., y E. Farjah, New control technique for compensation of neutral current harmonics in threephase four-wire systems, Conference IEEE Powertech, 1-6, (2009). 
